\title{
Existem diferenças nas respostas perceptuais ao treinamento de força em membros superiores e inferiores?
}

Are there difference in perceptual responses to resistance training on upper- and lowerlimbs?

Existen diferencias em las respuestas perceptivas al entrenamiento de fuerza en miembros superiores e inferiores?

\author{
José V. M. Moiano Júnior ${ }^{1}$ \\ Alysson Enes ${ }^{2 *}$ \\ Gustavo Oneda ${ }^{1}$ \\ Daniel M. de Cristo Neto ${ }^{2}$ \\ Ragami Chaves Alves ${ }^{2}$ \\ Diogo Palumbo' \\ Tácito Pessoa de Souza-Junior ${ }^{2}$ \\ Raul Osiecki ${ }^{1}$
}

\section{Resumo}

O objetivo do estudo foi comparar as respostas perceptuais ao treinamento de força em membros superiores e inferiores. Doze mulheres (idade $=23.8 \pm 5.1$ anos; massa corporal $=60.1 \pm 8.2 \mathrm{~kg}$; percentual de gordura $=23.5 \pm 4.7 \%) \mathrm{com}$ experiência prévia em treinamento de força foram recrutadas e submetidas a três sessões experimentais constituídas de séries de 5 repetições com 40\%, $60 \%$ e $80 \%$ de 1RM no supino, leg press, rosca direta e cadeira extensora, em dias diferentes. Após realização de cada série, a percepção de esforço foi coletada utilizando a escala OMNI-RES. Não foram encontradas diferenças estatisticamente significantes em relação as distintas naturezas biomecânicas dos exercícios, contudo, o esforço percebido foi estatisticamente significante quando se comparou as diferentes intensidades relativas no mesmo exercício. Portanto, não há diferenças nas respostas perceptuais entre membros superiores e inferiores. Em adição, a escala OMNI-RES foi uma ferramenta eficiente para controle de cargas fisiológicas (externas e internas) para membros superiores e inferiores em mulheres.

Palavras - chave: Esforço Físico. Percepção de Peso. Treinamento de Força. Cargas de treinamento.

\begin{abstract}
The aim of the study was to compare the perceptual responses to resistance training on upper- and lower-limbs. Twelve women (age $=23.8 \pm 5.1$ years; body mass $=60.1 \pm 8.2$ $\mathrm{kg}$; body fat $=23.5 \pm 4.7 \%$ ) with previous experience in resistance training were recruited and were submitted to a three training sessions of 5 sets with $40 \%, 60 \%$ and $80 \% 1 \mathrm{RM}$ on bench press, leg press, biceps curl and leg extension, on different days. After each complete set, the perceived effort was collected using the OMNI-RES scale. No statistically significances were found among biomechanical characteristics of exercises, however, perceived effort values were statistically significant when relative intensities were compared in the same exercise. Thus, there are no differences in perceptual responses
\end{abstract}


between upper- and lower-limbs exercises. In addition, the OMNI-RES scale was an efficient tool for monitoring physiological training loads (external and internal) for upperand lower-limbs in trained women.

Keywords: Physical Exertion. Weight Perception. Resistance Training. Training loads.

\section{Resumen}

El objetivo del investigación fue comparar las respuestas perceptivas al entrenamiento de fuerza en miembros superiores e inferiores. Doce mujeres ( $\mathrm{edad}=23.8 \pm 5.1$ años; masa corporal $=60.1 \pm 8.2 \mathrm{~kg}$; porcentaje de grasa $=23.5 \pm 4.7 \%)$ con experiencia previa en entrenamiento de fuerza fueron seleccionado e sometidas a três sesiones experimentales de 5 repeticiones con $40 \%, 60 \%$ y $80 \%$ de $1 \mathrm{RM}$ em los ejercicios press de banca, prensa de piernas, curl de bíceps e extensión de piernas en silla, en diferentes días. Después de cada serie, el esfuerzo percibido se recolectado utilizando la escala OMNI-RES. No se encontraron diferencias significativas entre las intensidades impuestas y entre las diferentes naturalezas biomecânicas de los ejercicios, por lo tanto, no hay diferencias em las respuestas perceptivas entre los miembros superiores e inferiores. Además, la escala OMNI-RES és una herramienta eficiente para controlar las cargas fisiológicas (externas e internas) para las miembros superiores e inferiores en mujeres.

Palabras - clave: Esfuerzo Físico. Percepción del Peso. Entrenamiento de fuerza. Carga de entrenamiento.

\section{INTRODUÇÃO}

O treinamento de força para

mulheres ocupa a segunda colocação em

número de praticantes no Brasil,

perdendo apenas para a caminhada'.

Com sua importância relatada em

diversos estudos, o treinamento de força

é uma opção interessante quando se fala

acerca de qualidade de vida até

desempenho esportivo. Sua prescrição é

baseada, comumente, em resultados

oriundos de testes de uma repetição

máxima (1RM), e apresenta como

principais variáveis de controle a intensidade, ordem dos exercícios, tipos

de exercícios, tempo de recuperação, frequência, número de séries e repetições, bem como a velocidade do movimento $^{2-4}$.

O controle de carga do treinamento está intimamente ligado a testes de intensidade, tais como o teste de 1RM. Porém, para indivíduos sedentários, iniciantes, idosos ou pessoas com limitações articulares e/ou musculares esse tipo de teste pode apresentar limitações como a falta de 
conhecimento específico do movimento ou limitação funcional ${ }^{5,6}$. Além disso, é um teste ineficiente quando se refere à realidade prática de muitas academias, por necessitar de um profissional acompanhando por um período de tempo prolongado. Sob esta perspectiva, elucida-se a utilização de escalas de percepção de esforço (PE) como um método que possa auxiliar a medida, controle e prescrição de cargas fisiológicas (externas e internas) treinamento de força ${ }^{7-9}$.

Nesse sentido, as escalas de Percepção de Esforço (PE) vêm se mostrando eficientes preditoras de esforço físico para diversas modalidades, uma vez que avalia a intensidade do exercício de forma eficiente e com um baixo custo, além de reportarem a intensidade percebida no momento do teste, considerando todos os possíveis fatores intervenientes. Sabe-se que o envolvimento de diferentes características biomecânicas e grupamentos musculares podem afetar a
PE, o que pode impactar no monitoramento das cargas fisiológicas de treinamento. Dessa forma, pode ser um fator relevante que o profissional das ciências do esporte tenha que levar em consideração ao utilizar tal ferramenta ${ }^{10-}$ ${ }^{12}$.

Considerando os fatos expostos, a hipótese é que existem diferenças perceptuais para uma mesma intensidade relativa entre membros inferiores e superiores. Portanto, o objetivo do presente estudo foi comparar as respostas perceptivas de mulheres em intensidades relativas a 40,60 e $80 \%$ da carga máxima em diferentes exercícios para diferentes membros.

\section{MÉTODOS}

\section{Participantes e aspectos éticos}

Foram recrutadas por conveniência 12 mulheres (idade $=23.8$ \pm 5.1 anos; massa corporal $=60.1 \pm 8.2$ $\mathrm{kg}$; estatura $=165.8 \pm 8.0 \mathrm{~cm}$; percentual de gordura $=23,5 \pm 4,7 \%$ ), com experiência prévia de pelo menos um 
ano com rotinas de treinamento de força

e devidamente familiarizadas com os exercícios do estudo. Os critérios de exclusão foram: (a) autorrelato de lesões musculoesqueléticas; (b) autorrelato de uso de recursos ergogênicos farmacológicos adrenérgicos e/ou androgênicos; (c) autorrelato de suplementação com cafeína ou tamponantes Este estudo foi aprovado pelo Comitê de Ética e Pesquisa com Humanos da Universidade Federal do Paraná (Parecer 1.377.596, CAAE 50981215.3.0000.0102).

\section{Protocolo experimental}

A coleta foi realizada em cinco encontros com os avaliados. No primeiro encontro, os participantes passaram por instruções e familiarização com a escala OMNI-RES, em conjunto com uma avaliação antropométrica. No segundo encontro foram realizados os testes de Uma Repetição Máxima (1RM) nos exercícios propostos e nos três encontros posteriores foram realizados os testes com intensidades impostas, de
$40 \%, 60 \%$ e $80 \%$ de 1RM, com intervalos mínimos de 24 horas entre os testes. Para os testes, as cargas foram randomizadas de maneira que apenas uma das três intensidades prédeterminadas se repetisse no dia. Cada indivíduo realizou uma única série com cinco repetições de cada exercício por dia, e intervalo entre séries de 2 minutos. Nos intervalos foi coletada a PE por meio da escala OMNI-RES, durante todos os dias de coleta. Os avaliados foram orientados a não treinar e nem consumir cafeína e/ou outros suplementos alimentares 24 horas precedentes dos testes. $\quad$ A figura 1 apresenta o protocolo experimental do estudo.

\section{Avaliação antropométrica}

A massa corporal foi medida utilizando uma balança com resolução de 50g (modelo 2096, Todelo®, São Paulo, Brasil), a estatura foi definida a partir de um estadiômetro (modelo ES2030, Sanny®, com resolução de 0,1 cm, São Paulo, Brasil) e para aferição 
das dobras cutâneas foi utilizado um

adipômetro (Skinfold Caliper,

Harpenden ${ }^{\circledR}$, com resolução de $0,2 \mathrm{~mm}$,

Baty International, England), com procedimentos padronizados propostos por Heyward ${ }^{13}$. A densidade corporal foi estimada pelos protocolos de Jackson, Pollock \& Ward $^{14}$ e o percentual de gordura corporal determinado pela equação de $\operatorname{Siri}^{15}$.

\section{Teste de 1 Repetição Máxima}

O protocolo de teste de $1 \mathrm{RM}$ incluiu um aquecimento específico do exercício em uma série de oito repetições a cerca de $50 \%$ de $1 \mathrm{RM}$ estimado pelo participante, seguido de outra série de $70 \%$ de $1 \mathrm{RM}$ estimada de apenas três repetições. Após o aquecimento, os sujeitos iniciavam o teste de 1RM que consistiu na execução completa de um movimento do exercício proposto não sendo possível realizar uma segunda repetição. A quantidade de tentativas não excedeu ao total de cinco, e os intervalos entre séries, tanto do aquecimento quanto das tentativas de 1RM tinham duração de 2 a 4 minutos, garantindo completa recuperação entre as tentativas ${ }^{16}$. Foram realizados os testes de $1 \mathrm{RM}$ para os quatro exercícios do protocolo experimental, no mesmo dia ${ }^{17}$.

\section{Sessões de treinamento}

Durante as sessões de treinamento, foi utilizada a escala OMNI-RES ao final de cada exercício e de cada série, com o participante tendo o tempo que julgasse necessário, dentro do intervalo padronizado de 2 minutos, para responder. Ao final de cada série foi coletada PE dos avaliados utilizando a escala OMNI-RES ${ }^{18}$. As participantes foram familiarizadas com a escala no primeiro encontro. No dia do teste de 1RM, repetiram-se as explicações acerca da escala, e nas sessões remanescentes foram coletadas a PE, sendo solicitado que indicassem um valor que quantificasse seu esforço nos exercícios.

A ordem de execução, bem como a intensidade que o participante fosse realizar o exercício no dia, foram 
randomizadas, garantindo que, ao longo das três sessões experimentais, as mesmas realizassem cada exercício com cada intensidade proposta, repetindo apenas uma única intensidade relativa, mas não o exercício na mesma sessão de treinamento.

\section{Análise estatística}

Os resultados foram apresentados em estatística descritiva no formato Média \pm Desvio Padrão (DP). Para as análises foi utilizado o software SPSS 20.0 (IBM Corp., Armonk, NY) em que os dados tiveram sua normalidade testada por meio do teste de Shapiro-Wilk, ao qual os pressupostos de normalidade foram atendidos. As médias da percepção de esforço foram comparadas mediante de uma ANOVA two way com medidas repetidas, sendo as variáveis independentes o exercício $\mathrm{e}$ as intensidades relativas. Foi utilizado o teste parcial eta ao quadrado para cálculo do tamanho do efeito $\left({ }_{p} n^{\mathcal{F}}\right)$. Foi utilizado o post-hoc de Bonferroni para identificação de diferenças entre os exercícios e intensidades. O nível de significância estatística adotado foi $\mathrm{p}<0$, 05.

\section{RESULTADOS}

A Tabela 1 apresenta os valores da PE para cada exercício com diferentes intensidades relativas. Os resultados demonstram que ao esforço percebido, através da escala OMNIRES, não apresentou interações estatisticamente significantes (M de Box $=14,273 ; \mathrm{F}_{(3,4)}=1,413 ; \mathrm{p}=0,189,{ }_{p} n^{2}=$ 0,08) entre os exercícios analisados, inferindo que não há diferenças perceptuais entre membros superiores e inferiores em mulheres.

A análise estatística apresentou interações e diferenças significantes para a PE entre diferentes intensidades relativas para o mesmo exercício $(\mathbf{M}$ de Box $=14,273 ; \mathbf{F}_{(2,43)}=134,28 ; p=0,0001 ;$ $\left.{ }_{p} n^{2}=0,862\right) . \quad \mathrm{A}$ análise post-hoc apresentou diferenças entre todas as intensidades relativas para cada exercício [PE Supino (40\% vs 60\%: $\mathrm{p}=0,018$, 
$40 \%$ vs $80 \%: p=0,0001,60 \%$ vs $80 \%: p$

$=0,0001){ }_{p} n^{2}=0,579$; PE Rosca Direta

$(40 \%$ vs $60 \%: \mathrm{p}=0,001,40 \%$ vs $80 \%: \mathrm{p}=$

$0,0001,60 \%$ vs $80 \%: \mathrm{p}=0,001){ }_{p} n^{2}=$

0,559; PE Leg Press (40\% vs 60\%: p = $0,0001,40 \%$ vs $80 \%: \mathrm{p}=0,0001,60 \%$ vs 80\%: $\mathrm{p}=0,01{ }_{p} n^{2}=0,622$; PE Cadeira

Extensora (40\% vs 60\%: $\mathrm{p}=0,0001,40 \%$ vs $80 \%: \mathrm{p}=0,0001,60 \%$ vs $80 \%: \mathrm{p}=$ $\left.0,0001){ }_{p} n^{2}=0,680\right]$.

\section{DISCUSSÃO}

Este estudo comparou as respostas perceptivas de mulheres em intensidades relativas a 40,60 e $80 \%$ da carga máxima em diferentes exercícios para diferentes membros. A hipótese de que existem diferenças perceptuais para uma mesma intensidade relativa entre membros inferiores e superiores em mulheres previamente treinadas foi refutada. Os principais resultados do presente estudo foram que a PE entre exercícios em mulheres previamente treinadas não apresentaram interação nem diferenças significantes. Em adição, a PE foi distinta para cada intensidade relativa no mesmo exercício.

Partindo da dificuldade de selecionar uma intensidade adequada para o treinamento de força, Robertson et al. ${ }^{18}$ sugerem que a escala OMNI-RES pode ser uma boa ferramenta para a quantificação do esforço no treinamento de força. Os resultados encontrados no presente estudo corroboram com o estudo de Robertson et al. ${ }^{18}$, uma vez que a PE foi distinta para as diferentes intensidades relativas, sugerindo uma quantificação do esforço no treinamento de força.

Em comparação com o presente estudo, Monteiro et al. ${ }^{19}$ realizaram um trabalho utilizando-se da PE em uma amostra similar, contudo, não encontrando diferenças estatisticamente significantes no que se refere às respostas perceptuais em diferentes intensidades e grupamentos musculares. Naclerio et al. ${ }^{20}$ recrutaram 11 adultos jovens que realizaram sessões de treinamento durante 8 dias, com intervalo de 48 
horas entre as sessões, assim como no presente estudo, os autores utilizaram a escala OMNI-RES para determinar a PE do indivíduo durante cada repetição e em cada série. Foram encontradas diferenças significantes entre a PE inicial e a PE após três repetições, encontrando, assim, a utilidade da PE para controle do esforço no treinamento de força.

Se tratando de comparação da PE em diferentes exercícios, assim como feito no presente estudo, Glass et al. ${ }^{21}$ relatam que o esforço percebido também não se alterou em comparação com as diversas intensidades e exercícios analisados. Apesar da natureza biomecânica influenciar as respostas perceptuais, nossos dados não apresentaram diferenças significativas entre membros superiores e inferiores. Isso pode ser atribuído ao fato que o treinamento não foi realizado até a falha concêntrica, sendo o esforço fisiológico global similar a diferentes cargas externas $\operatorname{impostas}^{22,23}$.
Estudos prévios ${ }^{25,26}$ mostraram que o nível de treinamento interferiu diretamente nas respostas perceptivas ao treinamento de força, onde participantes mais experientes reportaram $\mathrm{PE}$ inferior a participantes menos experientes em condições similares, o que sustenta a hipótese do nível de treinamento da amostra do presente estudo interferir na PE. Sabendo que a amostra do presente estudo possuía experiência prévia com treinamento de força, é possível que essa variável justifique a ausência de diferenças significantes entre os exercícios. Sugere-se estudos utilizando uma amostra superior e uma maior variedade nas escalas utilizadas, no nível de treinamento da amostra, com a adição de exercícios realizados até a falha concêntrica, com mais variáveis que possam ser exploradas como marcadores bioquímicos de carga interna de treinamento, que auxiliem na melhor compreensão acerca da PE e o controle e monitoramento de cargas fisiológicas no treinamento de força. 


\section{CONCLUSÃO}

Em suma, os resultados

encontrados no presente estudo

mostram que não há diferenças nas

respostas afetivas para exercícios com

intensidades de 40, 60, e 80\%.. Em

adição, a escala OMNI-RES é uma boa

ferramenta para mensuração e controle

de cargas fisiológicas de treinamento,

mesmo em diferentes intensidades e

exercícios.

\section{REFERÊNCIAS}

1. VIGITEL. Vigilância De Fatores De Risco E Proteção Para Doenças Crônicas Por Inquérito Telefônico. Brasil Ministério da Saúde Secretaria de Vigilância em Saúde Vigitel Brasil 2014. Epub ahead of print 2015. DOI: 10.7749/citiescommunitiesterritories.dez 2012.025.ackn.

2. Kraemer WJ, Adams K, Cafarelli $\mathrm{E}$, et al. Progression models in resistance training for healthy adults. Med $S c i$ Sports Exerc 2002; 34: 364-380.

3. LeSuer DA, McCormick JH, Mayhew JL, et al. The accuracy of prediction equations for estimating 1-RM performance in the bench press, squat, and deadlift. The Journal of Strength \& Conditioning Research 1997; 11: 211213.

4. Tiggemann CL, Korzenowski AL, Brentano MA, et al. Perceived exertion in different strength exercise loads in sedentary, active, and trained adults. The Journal of Strength \& Conditioning Research 2010; 24: 20322041.

5. ADAMS KJ, Swank AM, Barnard KL, et al. Safety of maximal power, strength, and endurance testing in older African American women. The Journal of Strength \& Conditioning Research 2000; 14: 254-260.

6. Pollock ML, Carroll JF, Graves $\mathrm{JE}$, et al. Injuries and adherence to walk/jog and resistance training programs in the elderly. Medicine and science in sports and exercise 1991; 23: 11941200 .

7. Scherr J, Wolfarth B, Christle JW, et al. Associations between Borg's rating of perceived exertion and physiological measures of exercise intensity. European Journal of Applied Physiology. Epub ahead of print 2013. DOI: 10.1007/s00421-012-2421-x.

8. Gearhart JRF, Goss FL, Lagally KM, et al. Ratings of perceived exertion in active muscle during high-intensity and low-intensity resistance exercise. Journal of Strength and Conditioning Research 2002; 16: 87-91.

9. Lagally KM, Mccaw ST, Young GT, et al. Ratings of perceived exertion and muscle activity during the bench press exercise in recreational and novice lifters. Journal of Strength and Conditioning Research 2004; 18: 359364.

10. Gentil P, Fisher J, Steele J. A review of the acute effects and long-term adaptations of single-and multi-joint exercises during resistance training. Sports Medicine 2017; 47: 843-855.

11. Borresen J, Lambert MI. The quantification of training load, the training response and the effect on performance. Sports medicine 2009; 39: 779-795.

12. Tiggemann CL, Pinto RS, Kruel LFM. A percepção de esforço no treinamento de força. Revista brasileira de medicina do esporte, São Paulo: SBME Vol 16, n 4 (jul/ago 2010), p 301309.

13. Heyward V. ASEP methods recommendation: body composition assessment. Journal of Exercise Physiology Online; 4.

14. Jackson AS, Pollock ML, Ward 
A. Generalized equations for predicting body density of women. Medicine and Science in Sports and Exercise. Epub ahead of print 1980. DOI: 10.1249/00005768-198023000-00009.

15. William E. Siri. Body Composition From Fluid Spaces and Density: Analysis of Methods. Adv Biol Med Phy.

16. Brown LE, Weir JP. ASEP procedures recommendation I: accurate assessment of muscular strength and power. Journal of Exercise Physiology Online; 4.

17. Baechle TR, Earle RW. Essentials of strength training and conditioning. Human kinetics, 2008.

18. Alves IC, Nascimento TM, Mendonça JDS, et al. Efeito dos diferentes intervalos de recuperação na percepção subjetiva de homens e mulheres. Revista Brasileira de Educação Física e Esporte. Epub ahead of print 2017. DOI: 10.11606/18075509201700040759.

19. Robertson RJ, Goss FL, Rutkowski J, et al. Concurrent validation of the OMNI perceived exertion scale for resistance exercise. Medicine $\&$ Science in Sports \& Exercise 2003; 35: 333-341.

20. Monteiro W, Simão R, Farinatti P. Manipulacion en el orden de los ejercicios y su influencia sobre el número de repeticiones y la percepcion subjetiva del esfuerzo en mujeres entrenadas. Revista Brasileira de Medicina do Esporte 2005; 11: 146-150. 21. Naclerio F, Rodr`’iguez-Romo G, Barriopedro-Moro MI, et al. Control of resistance training intensity by the OMNI perceived exertion scale. The Journal of Strength \& Conditioning Research 2011; 25: 1879-1888.

22. Glass SC, Stanton DR. Selfselected resistance training intensity in novice weightlifters. Journal of Strength and Conditioning Research. Epub ahead of print 2004. DOI: 10.1519/R-12482.1.

23. Pritchett RC, Green JM, Wickwire PJ, et al. Acute and session
RPE responses during resistance training: Bouts to failure at $60 \%$ and $90 \%$ of 1RM. South African Journal of Sports Medicine; 21.

24. Looney DP, Kraemer WJ, Joseph MF, et al. Electromyographical and perceptual responses to different resistance intensities in a squat protocol: does performing sets to failure with light loads produce the same activity? The Journal of Strength \& Conditioning Research 2016; 30: 792-799.

25. Tiggemann CL. Comportamento da percepção de esforço em diferentes cargas de exerc $\{\backslash \backslash$ i\}cios de força em adultos sedentários, ativos e treinados.

26. Palumbo D de P, Dias YR, Enes $\mathrm{AN}$, et al. Comparison of perceived exertion between bodybuilders and active individuals in different exercises and intensities. Journal of Exercise Physiology Online.

27. Fink J, Schoenfeld BJ, Kikuchi $\mathrm{N}$, et al. Effects of drop set resistance training on acute stress indicators and long-term muscle hypertrophy and strength. The Journal of Sports Medicine and Physical Fitness 2018; 58: 597-605.

Informações do artigo /

Information of this article:

Recebido: 11/04/2020

Aprovado: 21/08/2020

Publicado: 28/12/2020

Received: 11/04/2020

Approved: 21/08/2020

Published: 28/12/2020

Contribuição dos autores

JVMMJ, AE, GO, DMCN, RCA e DP contribuíram para a concepção e condução do estudo, além da análise e interpretação dos dados, e preparação do manuscrito para submissão. TPS e RO contribuíram para o conteúdo intelectual do manuscrito, e aprovaram a versão final para submissão.

Agradecimentos

Os autores gostariam de agradecer as participantes que voluntariamente participaram do presente estudo. 
Financiamento

Não houve financiamento para condução do estudo.

Conflito de interesses

Os autores declaram não haver conflito de interesses.

Como citar esse artigo / How to cite this article:

Moiano Júnior, J. V. M. et al. Existem diferenças nas respostas perceptuais ao treinamento de força em membros superiores e inferiores? Arq. Bras. Ed. Fís., Tocantinópolis, v. 3, n. 2, Ago./Dez., p. 39 49, 2020.

\section{Tabelas e figuras}

Tabela 1 - Percepção de esforço (PE) de mulheres jovens conforme os exercícios e intensidades.

\begin{tabular}{ccccc}
\hline $\begin{array}{c}\text { Intensidade } \\
\text { relativa }\end{array}$ & PE Supino & $\begin{array}{c}\text { PE Rosca } \\
\text { Direta }\end{array}$ & PE Leg Press & $\begin{array}{c}\text { PE Cadeira } \\
\text { Extensora }\end{array}$ \\
\hline & & \multicolumn{2}{c}{ Média (DP) $^{\text {Pend }}$} & \\
\hline $\mathbf{4 0 \% 1 R M}$ & $4,83(1,52)^{\mathrm{b}, \mathrm{c}}$ & $5,25(2,09)^{\mathrm{b}, \mathrm{c}}$ & $3,92(1,67)^{\mathrm{b}, \mathrm{c}}$ & $3,92(1,50)^{\mathrm{b}, \mathrm{c}}$ \\
$\mathbf{6 0 \% 1 R M}$ & $6,92(1,97)^{\mathrm{a}, \mathrm{c}}$ & $6,92(1,97)^{\mathrm{a}, \mathrm{c}}$ & $6,42(1,88)^{\mathrm{a}, \mathrm{c}}$ & $6,33(1,72)^{\mathrm{a}, \mathrm{c}}$ \\
$\mathbf{8 0 \% 1 R M}$ & $8,67(0,98)^{\mathrm{a}, \mathrm{b}}$ & $8,67(0,98)^{\mathrm{a}, \mathrm{b}}$ & $7,75(1,48)^{\mathrm{a}, \mathrm{b}}$ & $8,33(1,30)^{\mathrm{a}, \mathrm{b}}$ \\
\hline
\end{tabular}

Fonte: Os autores. DP $=$ desvio padrão. ${ }^{a}$ : diferença significante $(\mathrm{p} \leq 0,01)$ para $40 \%$; ${ }^{\mathrm{b}}$; diferença significante $(\mathrm{p} \leq 0,01)$ para $60 \%$; ${ }^{\mathrm{c}}$ : diferença significante $(\mathrm{p} \leq 0,01)$ para $80 \%$. Abreviações: $\mathrm{PE}=$ percepção de esforço; $1 \mathrm{RM}=1$ repetição máxima.

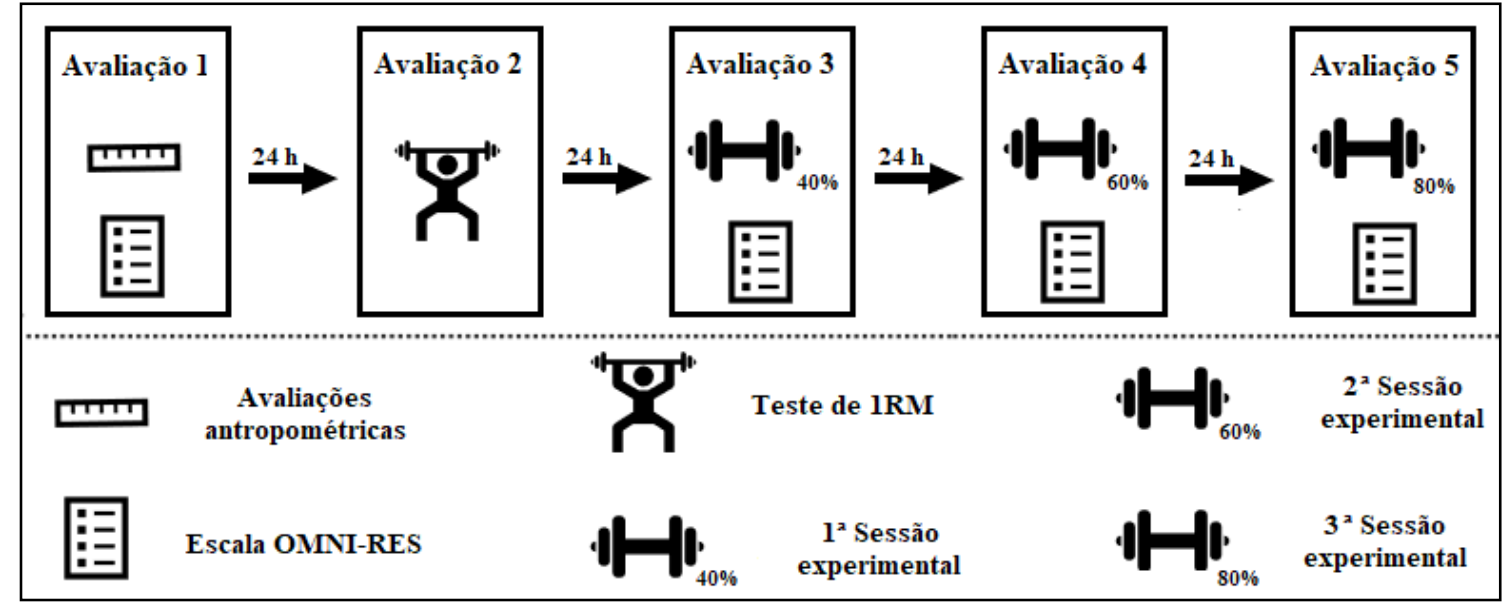

Figura 1 - Protocolo experimental 